Journal of

Epilepsy and

Clinical

Neurophysiology

J Epilepsy Clin Neurophysiol 2007; 13(4):163-167

\title{
Psicose e Depressão na Epilepsia do Lobo Temporal
}

\author{
Ludmyla Kandratavicius, Jaime Eduardo Cecílio Hallak, João Pereira Leite \\ Departamento de Neurologia, Psiquiatria e Psicologia Médica - Faculdade de Medicina de Ribeirão Preto
}

\begin{abstract}
RESUMO
Introdução: A epilepsia traz consigo um risco aumentado de desenvolvimento de distúrbios psiquiátricos. Pacientes com epilepsia parcial têm um risco maior do que aqueles com epilepsia generalizada idiopática. Além disso, a condição crônica das crises parece ser um fator importante no risco aumentado. Os sintomas resultam de mecanismos psicopatológicos muito diversos entre os pacientes. As classificações são baseadas na sintomatologia psiquiátrica, na presença ou ausência de distúrbios da consciência, em anormalidades do EEG e na relação temporal entre os sintomas e as crises. Resultados: Comorbidades psiquiátricas em epilepsia são muito freqüentes, especialmente aquelas que têm o lobo temporal como área epileptogênica principal. As desordens com maior prevalência são os distúrbios de humor como depressão maior, ansiedade e psicose. Conclusão: A epilepsia do lobo temporal per se, não pode ser considerada um fator de risco para o desenvolvimento de sintomas psiquiátricos, ou mais severos, dentre os pacientes com epilepsia parcial. Fatores concomitantes como duração da epilepsia e freqüência de crises podem ter um papel adicional importante. O reconhecimento mais acurado das comorbidades psiquiátricas poderá ajudar no desenvolvimento e implementação de programas apropriados de diagnóstico e tratamento, assim como melhorar o devir e a qualidade de vida dos pacientes com epilepsia.

Unitermos: epilepsia do lobo temporal, comorbidades psiquiátricas, psicose, depressão.
\end{abstract}

\begin{abstract}
Psychosis and depression in temporal lobe epilepsy

Introduction: Several studies have reported an increased risk for psychiatric disturbances in patients with epilepsy. It is assumed that the risk is higher for patients with partial epilepsy in comparison with patients with idiopathic generalized epilepsy. Besides, the persistence of seizures in some patients and the psychosocial impact of epilepsy seem to be important factors for the increased risk. The symptoms and the underlying psychopathology vary considerably among patients. Psychiatric comorbidities are based on the psychiatric symptomatology, the presence or absence of disturbance of consciousness, the EEG abnormalilies, and in the temporal relation between symptoms and seizures. Results: Psychiatric comorbidities in epilepsy are very common, especially in patients who have the temporal lobe as epileptogenic foci. Psychiatric disorders with a high prevalence in epilepsy include mood disorders such as major depression, anxiety, and psychosis. Conclusion: Temporal lobe epilepsy per se cannot be considered a risk factor in developing more or more severe symptoms of psychopathology in patients with partial epilepsy. Concomitant factors, such as the duration of epilepsy and seizure frequency may play an additional role. Better recognition of psychiatric comorbidities will help to develop and implement appropriate diagnostic and treatment programs, and improve functional outcomes and quality of life in people with epilepsy.
\end{abstract}

Key words: temporal lobe epilepsy, psychiatric comorbidities, psychosis, major depression. 


\section{EPILEPSIA E COMORBIDADES PSIQUIÁTRICAS}

Distúrbios psiquiátricos aparecem com freqüência muito maior em pacientes epilépticos do que na população em geral. A prevalência da presença de qualquer distúrbio psiquiátrico varia de 19 a 48\% entre pacientes epilépticos, com diferenças acentuadas entre os tipos de epilepsia considerados. ${ }^{1}$ Por exemplo, o risco de desenvolvimento de distúrbios psiquiátricos é maior em pacientes com ELT (60\%) e outras epilepsias focais (54\%), do que entre pacientes com epilepsia generalizada primária $(37 \%) .^{2}$

A depressão é tida como o aspecto mais comum que os acomete e a taxa de suicídio é de 9 a 25 vezes maior em pacientes com epilepsia. ${ }^{3}$ A prevalência de depressão varia de 20 a $55 \%$ em pacientes com crises recorrentes e de 3 a $9 \%$ em pacientes com crises bem controladas por medicamentos. ${ }^{4}$ Entre 25 e $52 \%$ dos pacientes epilépticos encaixam-se em critérios do DSM-IV para desordens de ansiedade. ${ }^{5}$ Já o risco para psicoses pode ser de 6 a 12 vezes maior em pacientes com epilepsia que na população em geral. ${ }^{6}$ Esquizofrenia e psicose schizophrenia-like podem afetar entre 4 e $18 \%$ dos pacientes epilépticos com epilepsia generalizada e ELT, respectivamente. ${ }^{1}$

Podemos classificar as comorbidades psiquiátricas das epilepsias em três grandes grupos: as síndromes ictais, as peri-ictais e as interictais. As síndromes ictais são aquelas que aparecem como uma crise parcial isolada ou como um componente de aura de uma crise parcial complexa. Por exemplo, em pacientes com crises epilépticas parciais, o medo é uma aura em cerca de $15 \%$ deles e é a mais comum emoção ictal - o que muitas vezes mimetiza ataques de pânico. ${ }^{7}$ Ao encontro destas ocorrências, temos que mais da metade de pacientes com desordens de pânico e sem história clara de crises epilépticas, quando monitorados por 24 horas, apresentam ondas de EEG epileptiformes.

As síndromes peri-ictais podem ser pré ou pós-ictais. Sintomas pré-ictais comuns incluem agitação psicomotora e confusão mental. As sídromes pós-ictais são aquelas relacionadas de forma mais direta com o período imediatamente após a crise até cerca de 72 horas. Neste intervalo, quando cansaço e confusão mental são sintomas comuns, podem também surgir sintomas de desordem afetiva e psicoses, que podem durar de algumas horas a meses, com intervalos de lucidez. Este tipo de síndrome está comumente associado a focos bilaterais e a aumento da freqüência das crises. ${ }^{9}$

As síndromes interictais caracterizam-se por episódios que se desenvolvem sem clara relação temporal com as crises epilépticas, de natureza crônica ou pelo menos mais duradoura, com sintomatologia que possa ser comparada a um evento psiquiátrico crônico não associado à epilepsia. ${ }^{10}$

\section{EPILEPSIA DO LOBO TEMPORAL}

A epilepsia do lobo temporal (ELT) é a forma mais comum de epilepsia focal em adultos, sendo responsável por $40 \%$ dos casos de epilepsia nesta população. ${ }^{11}$ Mais recentemente, a ELT foi subdividida em mesial e neocortical ou lateral, de acordo com a origem e semiologia das crises. Quanto a sua etiologia foi classificada em familiar, sintomática ou provavelmente sintomática. ${ }^{12}$

A epilepsia do lobo temporal mesial (ELTM), corresponde a aproximadamente $60 \%$ dos casos de ELT. ${ }^{13} \mathrm{~A}$ importância clínica da ELTM decorre de sua alta prevalência e elevada proporção de pacientes com crises epilépticas refratárias ao tratamento medicamentoso. ${ }^{14,15} \mathrm{~A}$ esclerose hipocampal (EH) é a etiologia encontrada em $50-70 \%$ dos pacientes com ELTM refratária ao tratamento clínico. ${ }^{16,17}$

Do ponto de vista anatomopatológico a esclerose hipocampal é caracterizada por perda neuronal e gliose, especialmente das células do setor de Sommer (CA1 e prósubículo) e dos neurônios da região hilar, com relativa preservação de CA2, subículo e giro denteado. ${ }^{18}$ Os setores de Spielmeyer $[\mathrm{CA} 3]^{19}$ e de Bratz $[\mathrm{CA} 4]^{20}$ também são relativamente bem conservados. Adicionalmente, observamos reorganização axonal, caracterizada por brotamentos de colaterais axônicos das células granulares (as fibras musgosas) na região da molecular interna do giro denteado. ${ }^{21,22}$

Apesar da relação entre EH e ELTM já estar bem estabelecida na literatura, a etiologia e o mecanismo exato pelo qual a EH participa da gênese das crises epilépticas ainda não foram completamente desvendados. Na década de $1950^{23}$ já especulavam que a EH estaria associada a uma história prévia de injúria precipitante inicial (IPI), tal como crises epilépticas, ocorrendo em fases precoces do desenvolvimento cerebral humano. A esclerose mesial seria provavelmente resultado de algum evento ocorrido no passado, e não conseqüência de convulsões repetidas.

Em 572 pacientes com ELTM intratável foi observada uma diminuição na densidade neuronal do hipocampo em pacientes com história longa de epilepsia. Entretanto, os autores enfatizaram que a perda neuronal só era vista em pacientes com história muito longa de doença (mais de 30 anos) e que a mesma era difusa, não se restringindo a subcampos específicos. Concluíram que crises límbicas ocorrendo por longas décadas contribuem na redução da população neuronal. No entanto, atribuíram à IPI o papel de promover dano neuronal capaz de produzir as condições necessárias para gerar crises epilépticas espontâneas. ${ }^{24}$

Associado à perda neuronal, observa-se também na EH a dispersão das células granulares. Esta é caracterizada pela perda da justaposição habitual das células granulares na camada granular do giro denteado, produzindo aumento de sua espessura e dos espaços intercelulares ${ }^{25}$. Em al- 
guns trabalhos foi observada a presença de distúrbios de migração das células piramidais e granulares, bem como alteração no padrão de laminação da formação hipocampal em camundongos mutantes. ${ }^{26}$ Esses dados sugerem a participação de fatores genéticos influenciando a fisiopatogenia da ELTM associada à esclerose hipocampal.

\section{PSICOSE EM EPILEPSIA}

A associação entre distúrbios psiquiátricos e epilepsia revista por Trimble, ${ }^{27}$ traz estudos de vários autores que estabelecem uma possível relação de antagonismo biológico entre psicose (especialmente os estados schizophrenialike) e epilepsia. Pacientes com demência ou distúrbios psiquiátricos que recebem drogas anticonvulsivantes tornamse mais deficientes em folato que pacientes epilépticos que recebem o mesmo tratamento. Assim, o folato é tido como convulsivante, considerando-se o fato que o déficit dele pode levar à diminuição de crises em presença de psicose. Este mesmo autor ${ }^{28}$ sugere que a dopamina (DA) também pode ter um envolvimento importante. Drogas antipsicóticas são comumente antagonistas de dopamina e podem provocar crises. Por outro lado, agonistas de DA aumentam os sintomas psicóticos ou podem desencadear psicose, e têm propriedades anticonvulsivantes. ${ }^{29-31} \mathrm{Uma}$ hipótese alternativa também pode ser construída com ácido gama-aminobutírico (GABA).

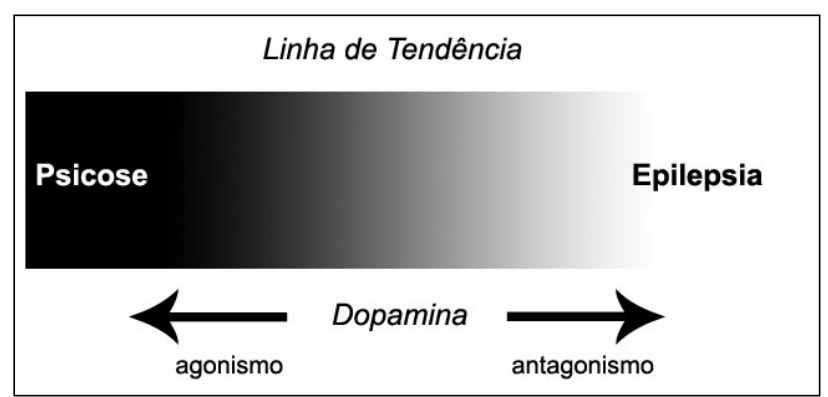

Figura 1. Relação entre epilepsia, psicose e dopamina (modificada de Trimble, 1991, p. 78).

A representação da Figura 1 não é universalmente aceita, já que em modelos experimentais de epilepsia utilizando ácido caínico, foi observado que antagonistas de DA tendem a reduzir a atividade das crises e da neurotransmissão excitatória. ${ }^{32}$

A associação entre epilepsia e distúrbios psiquiátricos tem sido observada desde a antiguidade. ${ }^{33}$ Desde o século XIX a resposta definitiva que justifique esta ligação ainda não foi anunciada. Podemos imaginar duas possibilidades distintas para a ligação: $1 \stackrel{-}{-}$ a associação entre epilepsia e distúrbios psiquiátricos é devida a uma combinação aleatória, seja por estatística tendenciosa, ou por combinação genética ao acaso que resulta em "piora genética" com o passar das gerações; $2 \stackrel{\circ}{-}$ a associação entre epilepsia e distúrbios psiquiátricos é real, já que epilepsia pode produzir psicose, ou psicose pode produzir epilepsia, ou ainda, que ambas resultem de um fator orgânico comum.

Um estudo recente de imagem comparando o fluxo sanguíneo regional cerebral de pacientes epilépticos com ausência e presença de psicose não detectou diferenças entre estes dois grupos na região do hipocampo. ${ }^{34}$ Entretanto, estudos com a proteína PSD95 (do inglês, post-synaptic density - PSD) e alguns subtipos de receptores NMDA têm resultados relevantes tanto para a epilepsia quanto para o estudo de distúrbios psiquiátricos. A PSD95 é conhecida por servir de ponte de ligação de várias moléculas ao receptor NMDA, entre elas, a sintase neuronal do óxido nítrico. ${ }^{35}$ Tal associação é normalmente deletéria, pois leva à ativação rápida da proteína p38 e conseqüente morte celular. ${ }^{36}$ Mas no hipocampo a PSD95 é uma das principais responsáveis pela formação dos complexos póssinápticos das fibras musgosas em CA3, mecanismo que é independente da ativação do receptor de glutamato. ${ }^{37} \mathrm{Com}$ relação aos distúrbios psiquiátricos, Toro e Deakin mostraram recentemente ${ }^{38}$ que a camada molecular do giro denteado de pacientes esquizofrênicos exibe menor expressão de PSD95 do que a mesma região hipocampal em pacientes com depressão.

\section{DEPRESSÃO EM EPILEPSIA}

A característica principal da depressão é o humor deprimido, ou perda de interesse e prazer por todas ou pela maior parte das atividades cotidianas ou do passado. Esta perturbação é proeminente, relativamente persistente e associada a outros sintomas da síndrome depressiva. Estes sintomas incluem distúrbios de apetite, mudanças no peso corporal, problemas de sono, agitação ou retardo psicomotor, energia diminuída, sensação de inutilidade ou culpa, dificuldade de concentração, pensamentos sobre morte e suicídio, ou tentativas de suicídio. ${ }^{39}$

A associação entre epilepsia e depressão é estudada desde Hipócrates, que escreveu: "Melancólicos ordinariamente tornam-se epilépticos, e epilépticos, melancólicos: o que determina a preferência é a direção que a doença toma; se recai sobre o corpo, epilepsia, se sobre a inteligência, melancolia". Como revisto recentemente por Kanner, ${ }^{40}$ esta proposição de cerca de 2400 anos tem se mostrado correta. $\mathrm{O}$ autor relaciona uma série de eventos comuns entre depressão e epilepsia, como a diminuição da atividade serotonérgica e noradrenérgica, além de anormalidades estruturais e funcionais no sistema límbico. Poderíamos esperar que pacientes com maior quantidade de crises teriam maior freqüência de episódios de depressão, quando comparados com pacientes com menos crises. Entretanto, a correlação entre freqüência de crises e gravidade da 
depressão parece não existir. Pacientes em estado livre de crises por no mínimo 6 meses continuaram a apresentar episódios de depressão. ${ }^{41}$ Em adição a isso, estudos variados com ratos Wistar geneticamente alterados para epilepsia do tipo ausência mostram que estes animais exibem acentuada imobilidade e ausência de comportamentos relacionados com prazer quando comparados a ratos Wistar não modificados geneticamente. ${ }^{42}$

Embora estudos de imagem funcional relatem hipoperfusão frontal em pacientes epilépticos deprimidos, ${ }^{43}$ há também estudos que relatam completa ausência de hipoperfusão frontal, e sim hiperperfusão temporal no hemisfério esquerdo em pacientes epilépticos deprimidos quando comparados com pacientes epilépticos sem depressão. ${ }^{44}$ É importante ressaltar que apenas no segundo estudo os pacientes foram selecionados com base em critérios diagnósticos para depressão e no qual a maioria das observações clínicas subjetivas sugeriam que o estado depressivo estava de alguma forma relacionado às crises.

\section{CONCLUSÃO}

Comorbidades psiquiátricas em pacientes com epilepsia parecem estar relacionadas ao quão crônica e severa é a epilepsia, e sua prevalência parece aumentar em pacientes com epilepsia refratária. Fatores endógenos como os genéticos, os efeitos das crises e a causa da epilepsia em si também podem influenciar a ocorrência das comorbidades psiquiátricas, além de fatores ambientais, efeitos psicossociais, de medicações, cirurgia, ou presença de uma comorbidade somática, como trauma craniano. Observações clínicas importantes nestes pacientes incluem localização do foco inicial da crise, lateralidade, tipo da neuropatologia, refratariedade e duração da epilepsia. Muitos dos pacientes com comorbidades psiquiátricas permanecem sub-diagnosticados e sem tratamento adequado e, embora não seja simples quantificar o efeito de uma condição psiquiátrica na epilepsia, é muito provável que ela resulte em piora significativa na qualidade de vida do paciente.

\section{AGRADECIMENTOS}

Os autores agradecem a todos os membros do Laboratório de Investigação em Epilepsias e às agências financiadoras FAPESP, CNPq, CAPES, FAEPA e PROEX pelos auxílios destinados a pesquisa e ensino.

\section{REFERÊNCIAS}

1. Gaitatzis A, Trimble MR, Sander JW. The psychiatric comorbidity of epilepsy. Acta Neurol Scand. 2004; 110:207-20.

2. Edeh J, Toone B. Relationship between interictal psychopathology and the type of epilepsy. Results of a survey in general practice. $\mathrm{Br}$ J Psychiatry. 1987; 151:95-101.

3. Kanner AM. The behavioral aspects of epilepsy: an overview of controversial issues. Epilepsy Behav. 2001; 2:8-12.

4. Gilliam FG et al. Depression in epilepsy: ignoring clinical expression of neuronal network dysfunction? Epilepsia. 2004; 45(Suppl 2):28-33.
5. Johnson EK, Jones JE, Seidenberg M, Hermann BP. The relative impact of anxiety, depression, and clinical seizure features on healthrelated quality of life in epilepsy. Epilepsia. 2004; 45:544-50.

6. Torta R, Keller R. Behavioral, psychotic, and anxiety disorders in epilepsy: etiology, clinical features, and therapeutic implications. Epilepsia. 1999; 40(Suppl 10):S2-20.

7. Young GB et al. Mesial temporal lobe seizures presenting as anxiety disorders. J Neuropsychiatry Clin Neurosci. 1995; 7:352-7.

8. Goldstein MA, Harden CL. Epilepsy and Anxiety. Epilepsy Behav. 2000; 1:228-34.

9. Devinsky O. Psychiatric comorbidity in patients with epilepsy: implications for diagnosis and treatment. Epilepsy Behav. 2003; 4(Suppl 4):S2-10.

10. Sachdev P. Schizophrenia-like psychosis and epilepsy: the status of the association. Am J Psychiatry. 1998; 155:325-36.

11. Engel Jr J. Introduction to temporal lobe epilepsy. Epilepsy Res. 1996; 26:141-50.

12. Engel Jr J. Classification of epileptic disorders. Epilepsia. 2001; 42:316.

13. Engel Jr J. Mesial temporal lobe epilepsy: what have we learned? Neuroscientist. 2001; 7:340-52.

14. Engel Jr J. Etiology as a risk factor for medically refractory epilepsy: a case for early surgical intervention. Neurology. 1998; 51:1243-4.

15. Stephen LJ, Kwan P, Brodie MJ. Does the cause of localisationrelated epilepsy influence the response to antiepileptic drug treatment? Epilepsia. 2001; 42:357-62.

16. Babb TL, Brown WJ. Neuronal, dendritic, and vascular profiles of human temporal lobe epilepsy correlated with cellular physiology in vivo. Adv Neurol. 1986; 44:949-66.

17. Leite JP et al. Plasticity, synaptic strength, and epilepsy: what can we learn from ultrastructural data? Epilepsia. 2005; 46(Suppl 5):134-41.

18. Sommer W. Erkrankung des Ammonshornsals aetiologisches Moment der Epilepsie. Arch Psychiatr. 1880; 10:631-75.

19. Spielmeyer W. Doe Pathogenese des epileptischen Krampfes. Z Dtsch Ges Neurol Psychiatr. 1927; 109:501-20.

20. Bratz E. Ammonschornbefunde der Epileptischen. Arch Psychiatr Nervenkr. 1899; 31:820-36.

21. Leite JP et al. Neuron loss, mossy fiber sprouting, and interictal spikes after intrahippocampal kainate in developing rats. Epilepsy Res. 1996; 26:219-31.

22. Babb TL, Kupfer WR, Pretorius JK, Crandall PH, Levesque MF. Synaptic reorganization by mossy fibers in human epileptic fascia dentata. Neuroscience. 1991; 42:351-63.

23. Meyer A, Falconer MA, Beck E. Pathological findings in temporal lobe epilepsy. J Neurol Neurosurg Psychiatry. 1954; 17:276-85.

24. Mathern GW, Adelson PD, Cahan LD, Leite JP. Hippocampal neuron damage in human epilepsy: Meyer's hypothesis revisited. Prog Brain Res. 2002; 135:237-51.

25. Houser CR. Granule cell dispersion in the dentate gyrus of humans with temporal lobe epilepsy. Brain Res. 1990; 535:195-204.

26. Lewis DV. Losing neurons: selective vulnerability and mesial temporal sclerosis. Epilepsia. 2005; 46(Suppl 7):39-44.

27. Trimble M. The psychosis of epilepsy. New York: Raven Press;1991.

28. Trimble M. The relationship between epilepsy and schizophrenia: a biochemical hypothesis. Biol Psychiatry. 1977; 12:299-304.

29. Ogren SO, Pakh B. Effects of dopamine D1 and D2 receptor agonists and antagonists on seizures induced by chemoconvulsants in mice. Pharmacol Toxicol. 1993; 72:213-20.

30. Turski L et al. Dopamine-sensitive anticonvulsant site in the rat striatum. J Neurosci. 1988; 8:4027-37.

31. Ben-Ari Y, Kelly JS. Dopamine evoked inhibition of single cells of the feline putamen and basolateral amygdala. J Physiol. 1976; 256:1-21.

32. Bourne JA, Fosbraey P, Halliday J. Changes in striatal electroencephalography and neurochemistry induced by kainic acid seizures are modified by dopamine receptor antagonists. Eur J Pharmacol. 2001; 413:189-98. 
33. Yacubian EM. Epilepsia: da antiguidade ao segundo milênio Saindo das sombras. São Paulo: Lemos; 2000.

34. Guarnieri R et al. Interictal SPECT in patients with mesial temporal lobe epilepsy and psychosis: a case-control study. Psychiatry Res. 2005; 138:75-84.

35. Kone BC. Protein-protein interactions controlling nitric oxide synthases. Acta Physiol Scand. 2000; 168:27-31.

36. Cao J et al. The PSD95-nNOS interface: a target for inhibition of excitotoxic p38 stress-activated protein kinase activation and cell death. J Cell Biol. 2005; 168:117-26.

37. Qin L, Marrs GS, McKim R, Dailey ME. Hippocampal mossy fibers induce assembly and clustering of PSD95-containing postsynaptic densities independent of glutamate receptor activation. J Comp Neurol. 2001; 440:284-98.

38. Toro C, Deakin JF. NMDA receptor subunit NRI and postsynaptic protein PSD-95 in hippocampus and orbitofrontal cortex in schizophrenia and mood disorder. Schizophr Res. 2005; 80:323-30.

39. Willner P. Depression - a Psychobiological Synthesis. New York: Willey Intercience; 1985.
40. Kanner AM. Depression in epilepsy: a neurobiologic perspective. Epilepsy Curr. 2005; 5:21-7.

41. Attarian H, Vahle V, Carter J, Hykes E, Gilliam F. Relationship between depression and intractability of seizures. Epilepsy Behav. 2003; 4:298-301.

42. Sarkisova KY, Midzianovskaia IS, Kulikov MA. Depressive-like behavioral alterations and $\mathrm{c}$-fos expression in the dopaminergic brain regions in WAG/Rij rats with genetic absence epilepsy. Behav Brain Res. 2003; 144:211-26.

43. Bromfield EB et al. Cerebral metabolism and depression in patients with complex partial seizures. Arch Neurol. 1992; 49:617-23.

44. Ring HA et al. Patterns of brain activity in patients with epilepsy and depression. Seizure. 1999; 8:390-97.

Endereço para correspondência:

Ludmyla Kandratavicius

Hospital das Clínicas da Faculdade de Medicina de Ribeirão Preto

Departamento de Neurologia, $4^{\circ}$ andar

Av. Bandeirantes, 3900

CEP 14049-900, Ribeirão Preto, SP, Brasi

E-mail:kandra@usp.br 